\title{
Ridge Polynomial Networks
}

\author{
Yoan Shin, Member, IEEE, and Joydeep Ghosh, Member, IEEE
}

\begin{abstract}
This paper presents a polynomial connectiontat network called ridge polynomial network (RPN) that can uniformly approximate any continuous function on a compact set in multidimensional input space $\Re^{d}$, with arbitrary degree of accuracy. This network provides a more efficient and regular architecture compared to ordinary higher-order feedforward networks while maintaining their fast learning property.

The ridge polynomial network is a generalization of the pisigma network and uses a special form of ridge polynomials. It is shown that any multivariate polynomial can be represented in this form, and realized by an RPN. Approximation copability of the RPN's is shown by this representation theorem and the Weierstrass polynomial approximation theonem. The RPN provides a natural mechanism for incremental network growth. Simulation results on a surface fitting problem, the classification of high-dimensional data and the realization of a multivariate polynomial function are given to highlight the the network. In particular, a constructive learatng ulgorithm developed for the network is shown to yield smooth generalization and steady learning.
\end{abstract}

\section{INTRODUCTION}

$\mathbf{S}$ EVERAL feedforward neural networks provide mapping capabilities for approximating reasonable functions. These include the multilayered perceptron (MLP) which can approximate reasonable functions to any desired degree of accuracy using only one hidden layer of units or nodes having sigmoid activation functions, provided that sufficiently many hidden nodes are available [6], [18]. Similarly, Park and Sandberg [29] recently showed that the radial basis function networks having one hidden layer are capable of universal approximation in the sense that they can approximate reasonable functions very well in terms of various metrics.

Another class of feedforward networks are the polynomial neural networks which use higher-order correlations of the input components to perform nonlinear mappings using only a single layer of units. Examples of polynomial neural networks include higher-order networks [17], sigma-pi networks [25], product units [8], and some functional link architectures [28]. The basic building block of higher-order networks is the $k$ th degree higher-order processing unit (HPU) with which a given

Manuscript received February 22, 1993; revised June 21, 1993 and October 11,1993 . This work was supported in part by ONR Contracts N00 014-89 C-0 298 and N00 014-92C-0232, by AFOSR Grant F49 620-93-1-0307, and by a faculty assistantship from TRW.

Y. Shin was with the Department of Electrical and Computer Engineering The University of Texas at Austin, Austin, TX and is now with the Department of Electonics Engineering, Soong Sil University, Seoul, South Korea.

J. Ghosh is with the Department of Electrical and Computer Engineering, The University of Texas at Austin, Austin, TX 78712 USA.

IEEE Log Number 9214275. function $f: \Re^{d} \rightarrow \Re$ is approximated as [17], [25]

$$
\begin{gathered}
f\left(x_{1}, \cdots, x_{d}\right) \approx \sigma\left(w_{0}+\sum_{i_{1}=1}^{d} w_{i_{1}} x_{i_{1}}+\sum_{i_{1}=1}^{d} \sum_{i_{2}=i_{1}}^{d}\right. \\
\cdot w_{i_{1} i_{2}} x_{i_{1}} x_{i_{2}}+\cdots \sum_{i_{1}=1}^{d} \sum_{i_{2}=i_{1}}^{d} \cdots \sum_{i_{k}=i_{k-1}}^{d} \\
\cdot w_{i_{1} i_{2} \cdots i_{k}} x_{i_{1}} x_{i_{2}} \cdots x_{i_{k}}
\end{gathered}
$$

where $\sigma(\cdot)$ is a suitable (linear or nonlinear) activation function, $x_{j}$ is the $j$ th component of input $\mathbf{x}=\left(x_{1}, \cdots, x_{d}\right) \in$ $\Re^{d}, w_{j k l \ldots} \in \Re$ is an adjustable weight (free parameter) from product of input components $x_{j}, x_{k}, x_{l}, \cdots$ to the output unit, and $w_{0} \in \Re$ is the threshold.' ${ }^{1}$ Since these networks do not have hidden layers, fast learning schemes such as Hebbian or LMS (least mean squares) type rules can be used. The approximation capabilities of this type of networks are obtainable from the Weierstrass polynomial approximation theorem. Unfortunately, the number of weights required to accommodate all higher-order correlations explodes with the input dimension, $d$ and the desired degree of the network, $k$. In fact, a $k$ th degree HPU needs a total of

$$
\sum_{i=0}^{k}\left(\begin{array}{c}
d+i-1 \\
i
\end{array}\right)=\left(\begin{array}{c}
d+k \\
k
\end{array}\right)
$$

weights if all products of up to $k$ components are to be incorporated. Consequently, typically only second or third order networks are considered in practice. Such a restriction to the order of the network leads to a reduction in the mapping capability, thereby limiting the use of this kind of higher-order networks.

To circumvent this problem, Shin and Ghosh [16], [34], [35] proposed a new class of polynomial feedforward neural networks called pi-sigma networks (PSN's) which use products of sums of input components instead of sums of products as in HPU's or "sigma-pi" units. The primary motivation was to develop a systematic method for maintaining the fast learning property and powerful mapping capability of single layer higher-order networks while avoiding the combinatorial increase in the number of weights required. A PSN is a feedforward network with a single "hidden" layer and product units in the output layer. Fig. 1 shows a PSN with single output. PSN's have a highly regular structure and need a much smaller number of weights compared to other single layer higher-order networks. The presence of only one layer

\footnotetext{
${ }^{1}$ In this paper, we interchangeably use $\mathbf{x}$ or $\left(x_{1}, \cdots, x_{d}\right) \in \Re^{d}$ to denote the input.
} 
of adaptive weights results in fast training. A given scalar function $f$ in $\Re^{d}$ is approximated by a $k$ th degree PSN as

$$
f\left(x_{1}, \cdots, x_{d}\right) \approx \sigma\left(\prod_{i=1}^{k} \sum_{j=1}^{d}\left(w_{i j} x_{j}+w_{i 0}\right)\right)
$$

where $w_{i j} \in \Re$ are adjustable weights. The total number of weights for a $k$ th degree PSN in $d$-dimensional input space is only

$$
(d+1) \cdot k \text {. }
$$

The price paid for using a fewer number of weights is that the PSN is not a universal approximator. In fact, a $k$ th degree PSN realizes a constraint representation of a $k$ th degree HPU. Empirical studies show, however, that it is a good model for smooth functions and gives good results on various applications [16], [34], [38].

In this paper, we present a polynomial connectionist network called ridge polynomial network (RPN) that has good mapping capabilities in the sense that any continuous function on a compact set in $\Re^{d}$ can be uniformly approximated by this network. The RPN is efficient in the sense that they utilize univariate polynomials which are easy to handle. This fact is contrary to the higher-order feedforward networks in which direct use of multivariate polynomials causes an explosion of free parameters. Also, this network leads to highly regular structure as compared to the ordinary higher-order networks while maintaining their fast learning property.

The RPN is a generalization of the PSN that uses a special form of ridge polynomials. A representation theorem is introduced to show that any multivariate polynomial can be represented in terms of this ridge polynomial, and realized by an RPN. Approximation capability of the RPN's is shown by this representation theorem and the Weierstrass polynomial approximation theorem. The RPN provides a natural mechanism for incremental network growth, and we develop a constructive learning algorithm for the network.

In the following section, the RPN is formulated [36], [37]. Its approximation capability is shown by using Chui and Li's representation theorem [3] and the Weierstrass polynomial approximation theorem. Also, we compare RPN with other polynomial approximation techniques, and discuss neurobiological plausibility of the RPN. In Section III, we develop a constructive learning algorithm for the RPN by which a parsimonious approximation of an unknown function in terms of network complexity can be achieved. Section IV presents simulation results on oceanic transient signal classification, a surface fitting problem and the realization of a multivariate polynomial using the constructive learning algorithm for the RPN. Section V concludes the paper.

\section{Ridge Polynomial Networks}

\section{A. Ridge Polynomials}

Let $C(K)$ be the vector space of continuous scalar functions on a compact subset $K$ of a Euclidean space $\Re^{d}(d \geq 1)$ such as a hypercube $[0,1]^{d}$. For $\mathbf{x}=\left(x_{1}, \cdots, x_{d}\right)$ and

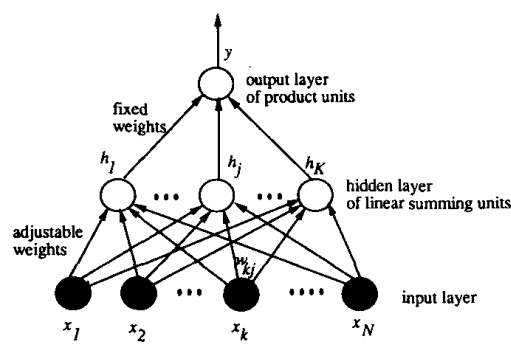

Fig. 1. A pi-sigma network with one output.

$\mathbf{w}=\left(w_{1}, \cdots, w_{d}\right) \in \Re^{d}$, we denote $\langle\mathbf{x}, \mathbf{w}\rangle$ as their inner product

$$
\langle\mathbf{x}, \mathbf{w}\rangle=\sum_{i=1}^{d} x_{i} w_{i}
$$

For a given compact set $K \subset \Re^{d}$, all functions defined on $K$ in the form of $f(\langle\cdot, \mathbf{w}\rangle): K \rightarrow \Re$, where $\mathbf{w} \in \Re^{d}$ and $f(\cdot): \Re \rightarrow \Re$ is continuous, are called ridge functions. A ridge polynomial is a ridge function that can be represented as

$$
\sum_{i=0}^{n} \sum_{j=1}^{m} a_{i j}\left\langle\mathbf{x}, \mathbf{w}_{i j}\right\rangle^{i}
$$

for some $a_{i j} \in \Re$ and $\mathbf{w}_{i j} \in \Re^{d}$. The following theorem by Chui and Li [3] states that any polynomial in $\Re^{d}$ can be expressed in terms of a ridge polynomial.

Theorem $I$ (Chui and Li [3]): Let $\Pi_{k}^{d}$ denote the set of all polynomials in $\Re^{d}$ with degree $\leq k$, and let $\mathbf{x}^{\mathbf{i}_{j m}}$ represent a polynomial in $d$ variables of total degree $j$, i.e., $\mathbf{x}^{\mathbf{i}_{j m}}=$ $x_{1}^{i_{j m 1}} \cdots x_{d}^{i_{j m d}}$, with $=i_{j m 1}+\cdots+i_{j m d}=j$ for any $m$. For any polynomial

$$
p(\mathbf{x})=\sum_{j=0}^{k} \sum_{m=1}^{n_{j}} c_{j m} \mathbf{x}^{\mathbf{i}_{j m}}
$$

in $\Pi_{k}^{d}$ where $c_{j m} \in \Re$, there exist constants $a_{j m} \in \Re$ and $\mathbf{v}_{j m} \in \Re^{d}$ such that

$$
p(\mathbf{x})=\sum_{j=0}^{k} \sum_{m=1}^{n_{j}} a_{j m}\left\langle\mathbf{x}, \mathbf{v}_{j m}\right\rangle^{j}
$$

where $n_{j}=\left(\begin{array}{c}d+j-1 \\ j\end{array}\right)$, is the total number of $j$ th degree polynomials in $d$ variables. That is, $\sum_{m=1}^{n_{j}} c_{j m} \mathbf{x}^{\mathbf{i}_{j m}}$ in (6) denotes all $j$ th order terms of $d$ input variables.

Basically, (6) means that $p(\cdot)$ is a polynomial of $d$ variables comprising of all the terms from a constant to $k$ th order terms of $d$ variables. The original form of the theorem is more complicated than the one presented here. In the original theorem, constants $a_{j m}$ are uniquely determined from the linear equations. Since we are only concerned with the existence of a representation of multivariate polynomials in terms of ridge polynomials, however, a simpler statement is adopted.

Based on Theorem 1, we formulate a more general and efficient ridge polynomial. The result is presented in the following theorem and a detailed proof can be found in Appendix A. The theorem implies that a new polynomial 
structure (i.e., ridge polynomial network) can be formed by addition of PSN's of different degrees, and this new structure has the same approximation capability as that of ordinary multivariate polynomials. The proof is obtained by showing that the ridge polynomial given by the right-hand side of (7) is a special case of the RPN.

Theorem 2: For any polynomial

$$
p(\mathbf{x})=\sum_{j=0}^{k} \sum_{m=1}^{n_{j}} c_{j m} \mathbf{x}^{\mathbf{i}_{j m}}
$$

in $\Pi_{k}^{d}$ where $c_{j m} \in \Re$, there exist $w_{j i} \in \Re$ and $\mathbf{w}_{j i} \in \Re^{d}$ such that

$$
p(\mathbf{x})=\sum_{j=1}^{N} \prod_{i=1}^{j}\left(\left\langle\mathbf{x}, \mathbf{w}_{j i}\right\rangle+w_{j i}\right)
$$

where $N=\sum_{l=0}^{k} n_{l}$.

The uniform approximation capability of the ridge polynomials which have the form of (8) is immediate.

Proposition 1: Let $f \in C(K)$ and $\epsilon>0$ be given. Then there exists a ridge polynomial $p \in C(K)$ of the form (8) such that $|f(\mathbf{x})-p(\mathbf{x})|<\epsilon$ for all $\mathbf{x} \in K$.

Proof: By the generalized Weierstrass polynomial approximation theorem [7] (see Appendix B), there is a polynomial $p \in C(K)$ of the form (6) such that $|f(\mathbf{x})-p(\mathbf{x})|<\epsilon$ for all $\mathrm{x} \in K$. Thus, the result follows from Theorem 2 .

\section{B. Definition of Ridge Polynomial Networks}

A ridge polynomial network (RPN) is defined as a feedforward network based on (8). An unknown function $f$ defined on a compact set $K \subset \Re^{d}$ can be approximated by the RPN as

$$
\begin{aligned}
f(\mathbf{x}) \approx & \left(\left\langle\mathbf{x}, \mathbf{w}_{11}\right\rangle+w_{11}\right)+\left(\left\langle\mathbf{x}, \mathbf{w}_{21}\right\rangle+w_{21}\right) \\
& \cdot\left(\left\langle\mathbf{x}, \mathbf{w}_{22}\right\rangle+w_{22}\right)+\cdots+\left(\left\langle\mathbf{x}, \mathbf{w}_{N 1}\right\rangle+w_{N 1}\right) \cdots \\
& \cdot\left(\left\langle\mathbf{x}, \mathbf{w}_{N N}\right\rangle+w_{N N}\right)
\end{aligned}
$$

Note that each product term in (9) is obtainable as the output of a pi-sigma network (PSN) with linear output units. Thus, this new formulation can be considered to be a generalization of the PSN's. The total number of coefficients or weights in (7) and (8) is $\mathcal{O}(d \cdot N)$ and $\mathcal{O}\left(d \cdot N^{2}\right)$, respectively. In terms of total number of weights, (8) is less efficient than (7). The more regular and systematic structure of (8) over (7), however, makes it a more suitable basis for a feedforward network. Since, in general, there may not be much a priori information about the function to be approximated, it is difficult to choose an appropriate network size based on (7). On the other hand, an RPN based on (8) can be incrementally grown to meet a predefined error criterion. This idea is discussed again in the following section.

The RPN is closely related to a statistical regression technique called projection pursuit regression [20]. Loosely speaking, projection pursuit aims to systematically find "good" low dimensional projections of a high dimensional data points by optimizing a certain performance objective function. Projection pursuit is a powerful method which can avoid the curse of dimensionality [20] by working on low dimensional projections. A projection pursuit technique typically uses ridge functions to approximate a multivariate function $f$ by forming a summation (projection pursuit regression [12]) or product (projection pursuit density estimation [13]). The basic formulation of projection pursuit regression is as follows

$$
f(\mathbf{x}) \approx \sum_{i=1}^{k} a_{i} g_{i}\left(\left\langle\mathbf{x}, \mathbf{w}_{i}\right\rangle\right)
$$

for some continuous functions $g_{i}$ in one variable, and $a_{i} \in$ $\Re, \mathbf{w}_{i} \in \Re^{d}$. The determination of $g_{i}$ is usually done by one-dimensional data smoothing over a certain bandwidth of projection, and $a_{i}$ and $\mathbf{w}_{i}$ is usually determined by optimizing certain performance indexes. Projection pursuit is a good example where parameterized $\left(a_{i}, \mathbf{w}_{i}\right)$ and nonparameterized $\left(g_{i}\right)$ methods are combined. Researchers have shown that projection pursuit networks based on (10) work better on angular smooth functions than on Laplacian smooth functions [45]. Also, a learning rule taken from projection pursuit regression literature has been compared with backpropagation for training MLP networks [19]. In these studies, the projection pursuit learning achieves more parsimonious approximation (i.e., fewer number of hidden units for a given approximation accuracy) for many kind of nonlinear functions as compared to the standard backpropagation learning for the MLP.

The RPN and projection pursuit are similar in spirit. In fact, (8) is considered as a special case of projection pursuit regression with fixed functions $g_{i}$. Since projection pursuit finds the kernels $g_{i}$ through more data pertinent smoothing methods, it realizes more parsimonious structure as compared to the RPN which uses fixed polynomial functions. The kernels $g_{i}$ need to be obtained by nonparameterized methods in projection pursuit, however, which is not the case in RPN.

\section{Network Architecture}

Neural network architectures are either fixed or dynamic in size. In a fixed-size architecture, the size of an instance of a network, in terms of number of units and weights, does not change from its first construction. On the other hand, in a dynamic-size architecture, the size of a network can be changed during the learning process. In particular, a constructive learning (incremental learning) method grows the network structure from a small, basic network for a given problem and the network becomes larger as learning proceeds until the desired level of approximation error is attained. The "cascade-correlation learning" [9] is an example of the constructive learning method. The main advantage of a constructive learning algorithm is that the networks realize a parsimonious approximation of the problem, typically resulting in better generalization.

Fig. 2 shows a generic network architecture of the RPN using PSN's as basic building blocks. Note that the RPN has only a single layer of adaptive weights which has benefits in terms of training speed. Instead of a linear activation function, we can use a nonlinear function such as sigmoid or hyperbolic tangent, in practical applications. Thus, an unknown scalar 


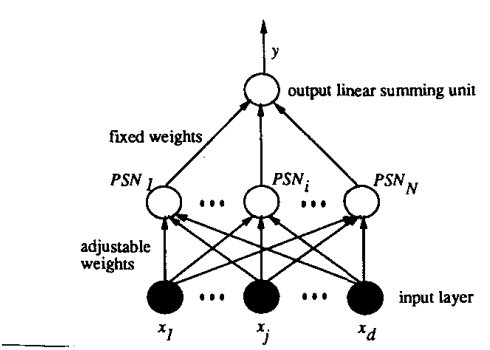

Fig. 2. A ridge polynomial network. Each $P S N_{i}$ represents an $i$ th degree pi-sigma network with linear output activation function.

function $f$ in $\Re^{d}$ can be approximated by the direct use of the RPN of degree up to $k$ based on

$$
f(\mathbf{x}) \approx \sigma\left(\sum_{i=1}^{k} P_{i}(\mathbf{x})\right)
$$

where

$$
P_{i}(\mathbf{x})=\prod_{j=1}^{i}\left(\left\langle\mathbf{w}_{j}, \mathbf{x}\right\rangle+w_{j 0}\right), \quad i=1, \cdots, k .
$$

In (12), $\sigma(\cdot)$ is a suitable (linear or nonlinear) activation function and $\mathbf{w}_{j} \in \Re^{d}$ and $w_{j 0} \in \Re$ are determined by any suitable learning process. A constructive learning algorithm based on gradient descent is given in Section III.

\section{Relation to Other Polynomial Approximation Techniques}

Several types of higher-order networks and polynomialbased approximation techniques can be found in neural network, system theory and estimation theory literature. In this subsection, we summarize notable alternatives and compare them with RPN's to highlight key differences.

1) Feedforward Networks with Product Units and Static Architectures: Besides the HPU networks, this category includes several models in artificial neural network and system theory that are closely related. One such model is the "sigma-pi unit" [25], first used in a connectionist framework by Feldman and Ballard [10]. Since in this model, sums of products of selected input components are utilized, these units are a special case of HPU's. Since this model does not allow terms like $x_{i}^{k}(k>1)$, however, a single layer of sigma-pi units is not capable of approximating some functions well [5].

To counter the drawbacks of sigma-pi units, Durbin and Rumelhart [8] proposed the use of product units, each of which could realize a single higher order term, i.e., the output $z_{j}: \Re^{d} \rightarrow \Re$ of the $j$ th product unit is given by

$$
z_{j}\left(x_{1}, \cdots, x_{d}\right)=\prod_{i=1}^{d} x_{i}^{p_{j i}}
$$

The novelty lay in the fact that the exponents $p_{j i}$ s could also be adapted using gradient descent, and were not limited to integer values. The authors envisioned that the product units would be mixed with summing units to form weighted sums of arbitrary products, and focused on networks with a single hidden layer of product units, with output $y: \Re^{d} \rightarrow \Re$ given by

$$
y\left(x_{1}, \cdots, x_{d}\right)=\sum_{j} w_{j} z_{j}\left(x_{1}, \cdots, x_{d}\right)
$$

with $w_{j} \in \Re$. The resulting networks are efficient for problems where a suitable discriminating function can be easily expressed in a (real-valued) sum of products form. For example, if each input component is \pm 1 , parity is simply obtained by checking whether the product of all the input components is +1 or not. As expected, product units are efficient for parity and symmetry problems.

The application of product unit networks to continuous valued problems has not been investigated much, either in [8] or in later works. We note that their success depends on whether the number of product units chosen is appropriate or not. This number may have little to do with the order of the function. Also, a network based on (14) is slowed because of backpropagation of error. In contrast, in the constructive learning scheme for the RPN which will be described in Section III, PSN's of increasing degrees are sequentially added during training until a desired level of performance is obtained. Thus little a priori knowledge of the desired function is required. Also, the RPN has only a single layer of adaptive weights as in the HPU.

The functional link architecture has also been proposed for generating higher-order or nonlinear functions of the input components [28]. A functional link architecture estimates an unknown function as $\sum_{i} w_{i} \phi_{i}(\cdot)$, where $\phi_{i}(\cdot)$ s are input components, products of input components, or other suitable functions such as sinusoidal functions. This approach is essentially linear regression, with $\phi_{i}(\cdot)$ s serving as the basis functions, and has been well studied in several disciplines. In [28], excellent results are obtained for the three-parity problem by choosing $x_{1}, x_{2}, x_{3}, x_{1} x_{2}, x_{1} x_{3}, x_{2} x_{3}$, and $x_{1} x_{2} x_{3}$ as the basis functions. Clearly, a priori knowledge has been used to choose such an optimal basis. In another example on approximating a "sinusoidal" surface, the basis used was $x, \sin (\pi x), \cos (\pi x), \cdots$ and not a single higher-order polynomial was used. Surely this basis would not work well for the parity problem. Thus the functional link network does not answer the key issue of how to determine a suitable basis for arbitrary functions. As mentioned earlier, the issue when using RPN is to select suitable thresholds for adding another PSN, which is much simpler. Thus even the order may not be selected a priori, and rather be determined by the data.

In system identification theory, (1) is identical to a Gabor-Kolmogorov polynomial expansion for a system [24], provided a linear activation function $\sigma(\cdot)$ is used. Here, the goal is to achieve polynomial approximation of the function using this model. A study of polynomial discriminant functions based on (1) with a thresholding transfer function, can also be found in classical pattern recognition literature [39]. Such functions have been revisited by Psaltis et al.; [30] who study the increase in memory capacity because of the presence of more degrees of freedom, and propose optical implementations. Since RPN's are founded on (8), they are closer to projection pursuit regression, as discussed in Section II-B and 
have properties that are quite different from approaches based on (1) when the order of approximation is finite.

2) Incremental Polynomial Approximation: An alternative to parametric approaches is to use incremental techniques in which the number of free parameters is gradually increased, if need be. Ivakhnenko has proposed a polynomial approximation algorithm, called the group method of data handling (GMDH) algorithm, for complex nonlinear systems [21]. This algorithm is basically an incremental algorithm for building an approximation of the unknown system by beginning with simple polynomials and proceeding to more and more complex polynomial expressions. Its functioning can be expressed in terms of the incremental construction of a feedforward network in which the output of each node is a product of two variables and whose parameters are obtained by regression. Starting with the input nodes, nodes are created in the next layer by pairwise connecting the inputs of the previous layer. The power of the method arises from the use of simple lowdimensional functions and the ability of the algorithm to discard nodes with little promise. A heuristic that evaluates how close the realized function describes the training data in a least-mean-squares sense is used to terminate and evaluate the algorithm. Unfortunately, this approach often leads to suboptimal structures because of its heuristic nature.

Another similar approach is the self-organizing neural network (SONN) algorithm [40]. This algorithm constructs a network, chooses the activation functions, and adjusts the weights to incrementally build a model of the unknown system using representations selected from a predetermined set of polynomials. The algorithm shares the spirit of GMDH type algorithms, but the use of a modified minimum description length criterion in conjunction with stochastic search based on simulated annealing for selection of node transfer functions leads to models that are simpler and more accurate. Comparisons with MLP given in [40] for the Mackey-Glass chaotic time series prediction problem show that the number of epochs required is less by an order of magnitude. This advantage is more than offset by the long times taken per epoch, largely due to simulated annealing search, which also degrades rapidly in time and quality with increasing problem size.

The RPN is similar to GMDH and SONN in that the network is based on higher-order polynomials and is grown incrementally. Both GMDH and SONN lead to networks with arbitrary number of hidden layers and nodes in contrast to RPN's which have a well regulated architecture. Besides this difference and the difference in representation forms, the main trade-off is that RPN does not require extensive preprocessing using training data to come up with the desired structure, but the generality of the RPN structure also indicates that it may not be optimized for specific problems. Experimental results show that RPN's perform well over a broad class of problems, but may not be the best for a specific problem.

The LMS tree algorithm proposed by Sanger [32] also falls in this category if used with polynomial bases. The LMS tree starts with basis functions for an input component, and grows a "subnetwork" under the basis function which has the maximum error variance. The subnetwork uses basis functions for a different input component, and thus successively forms basis functions which are factorized with respect to each input coordinate. Sanger $e t$ al. also proposed an iterative construction algorithm of sparse polynomial approximation which can be used in multidimensional input space [33]. The algorithm constructs a polynomial approximation of a given function from lower to higher order by "looking-ahead" relevant terms which will maximally contribute to decreasing the error if added. This algorithm is based on the LMS tree and a new figure-of-merit called "potential" is used for the look-ahead mechanism. These algorithms are different from the incremental algorithm of the RPN in the sense that they use different polynomial structures (factorized polynomials in the LMS tree and ridge polynomials in the RPN) and network growing algorithms. They also need extra processing for network growing mechanism. As in the case of GMDH and SONN, these algorithms yield irregular and problem-specific network structure as compared to RPN's highly regular structure.

3) Higher-Order Correlations in Recurrent Nets: The use of polynomial threshold elements of degree $d(d \geq 2)$ has been considered in the context of Hopfield-type networks [42]. An extension of the outer-product storage rule to incorporate higher order correlations has also been proposed [2]. These studies pertain to fully connected recurrent networks and are mainly concerned with increasing storage capacity when such nets are used as associative memories. Thus they are fundamentally different from RPN's in structure and purpose, and are not considered any further.

\section{E. Neurobiological Plausibility of RPNs}

Are RPN type computing structures even remotely neurobiologically plausible? In neurophysiology, the possibility that dendritic computations could include local multiplicative nonlinearities is widely accepted [27]. Indeed, Durbin and Rumelhart [8] observe that there is a natural neurobiological interpretation of a combination of product and summing units in terms of a single neuron or a group of neurons. In particular, local regions of dendritic arbor could act as product units whose outputs are summed at the soma. Mel and Koch have also argued that sigma-pi units underlie the learning of nonlinear associative maps in cerebral cortex [27], and that aggregates (on the order of 100 cells) of pyramidal cells whose apical dendrites rise toward the cortical surface in tight clumps, act as a single virtual sigma-pi unit. A recent review of a number of different biophysical mechanisms that give rise to multiplicative interactions can be found in [22]. These mechanisms range in specificity of interactions from pairs of individual synapses to small sets of neurons.

Mel has recently proposed the clusteron as an abstraction for a complex neuron that can extract higher order statistics from input stimuli [26]. In his model, a dendritic tree receives weighted synaptic contacts from a set of afferent axons. Each synaptic contact is given by a product of direct stimulus intensity and a weighted sum of neighborhood activity. We note that this description translates to an RPN, which can be considered as a mathematical abstraction of the clusteron model. Certainly, however, a biologically plausible network should have some self-organizing capabilities. This motivates 
investigation of Hebb-type learning schemes for the RPN to further pursue the plausibility issue.

\section{LEARNING ALGORITHM FOR THE RPN}

Since each $P_{i}$ in (11) is obtainable as the output of a PSN of degree $i$ with linear output units, the learning algorithms developed for the PSN can also be used for the RPN's. So an immediate approach is to select the constituent PSN's and then adapt weights using gradient descent. The optimal network complexity, however, as determined by $k$, is often not known a priori. This has prompted several constructive (or destructive) algorithms that dynamically adapt network structure by adding (or pruning) weights or nodes until the most appropriate network size is obtained [41]. The tradeoffs involved in network size vs. generalization capability can be understood in terms of the bias/variance dilemma [14]. As a network grows, model bias is decreased resulting in better generalization. If the number of free parameters is too large, however, model variance dominates and starts to degrade generalization performance. These considerations motivate us to develop a constructive learning algorithm for the RPN's in this section. This learning algorithm aims at approximating an unknown scalar function $f$ in $\Re^{d}$ by successive addition of a PSN, $P_{i}$ of higher degree than previously incorporated ones until a desired level of accuracy is attained.

\section{A. Adaptation of Weights in Pi-Sigma Networks}

Before presenting the constructive learning algorithm for the RPN, we summarize the learning algorithms for the PSN which are a basic building block of the constructive learning algorithm for the RPN.

Learning algorithms for PSN's are based on gradient descent on the estimated mean squared error surface in weight space. For a PSN, the mean squared error (MSE) objective is as follows

$$
\epsilon=\sum_{p=1}^{L} \epsilon^{p}=\frac{1}{L} \sum_{p=1}^{L}\left(f^{p}-y^{p}\right)^{2}
$$

where $f$ is the unknown desired function to be approximated, superscript $p$ denotes the $p$ th training pattern, $f^{p}$ is the desired output for the $p$ th pattern, $y^{p}=\sigma\left(\prod_{j} h_{j}^{p}\right)$, and the summation is over all $L$ training patterns. ${ }^{2}$

In typical feedforward networks, all the weights are modified at each update step. This updating scheme can lead to instability problems for the PSN, however, unless the learning rate is sufficiently small. This is due to higher order crossterms generated at the output product unit that may not be in the direction of steepest gradient. In [16], we formally show why the magnitude of the learning rate is limited by a fully synchronous updating scheme. Instead of modifying all the weight sets synchronously at each update step, one subset of weights chosen at a time for updating. We proposed

\footnotetext{
${ }^{2}$ For simplicity, a one-dimensional output is considered. If multiple outputs are needed, the summation is also performed over all outputs and the final value is also divided by the number of output units to get the error measure. The extension is fairly straightforward, so we will concentrate only on the single output case.
}

the randomized [34] and asynchronous updating rules [35] as methods to choose weight sets to be updated.

- Randomized Rule: Consider a PSN with $d$ inputs and $k$ summing units. At each update step, we randomly select a summing unit and only update the set of $d+1$ weights associated with its inputs.

- Asynchronous Rule: At each update step, all $k$ sets of weights are updated but in an asynchronous manner. That is, one set of weights

$$
\mathbf{v}_{j}=\left(w_{j 0}, \mathbf{w}_{j}\right)=\left(w_{j 0}, w_{j 1}, \cdots, w_{j d}\right)
$$

(corresponding to the $j$ th summing unit) is chosen at a time and modified according to the weight update rule. Then, for the same input pattern, the output is recomputed for the modified network, and the error is used to update a different set of weights. For every input, this procedure is performed $k$ times so that all $k$ sets of weights are updated once.

The improved properties of both of these approaches is explained by the analysis given in [16]. In this paper, we concentrate on the asynchronous rule which performs better than the randomized one in terms of training speed.

After the set of weights $\mathbf{v}_{j}$ to be updated is chosen by any of above rules, an LMS-type approach is taken [44]. Here, the instantaneous squared error for the $p$ th pattern $\epsilon^{p}$ is used as an estimate of the true MSE. Applying gradient descent on the selected set of weights $v_{l}$, one obtains

$$
\Delta w_{l i} \propto-\frac{\partial \epsilon^{p}}{\partial w_{l i}}, \quad i=0,1, \cdots, d
$$

leading to the following update rule

$$
\Delta \mathbf{v}_{l}=\eta \cdot\left(f^{p}-y^{p}\right) \cdot\left(y^{p}\right)^{\prime} \cdot\left(\prod_{j \neq l} h_{j}^{p}\right) \cdot \mathbf{z}^{p}
$$

where $\left(y^{p}\right)^{\prime}$ is the first derivative of the output, $\mathbf{z}=(1, \mathbf{x}) \epsilon$ $\Re^{d+1}$ is the augmented input pattern and $\eta$ is the learning rate. Other sets of weights which are not selected remain the same. The methods are easily extensible for multiple outputs since each output has its own summing units.

\section{B. Constructive Learning Algorithm for the RPN}

As discussed earlier, the constructive learning algorithm for the RPN is based on the asynchronous update rule for the PSN. Before presenting the algorithm, we need the following notations- $\epsilon_{t h}$ : threshold mean squared error (MSE) for the training phase, $\epsilon_{c}, \epsilon_{p}$ : MSE's for the current epoch and previous epoch during the training, respectively, $r$ : threshold for successive addition of a new PSN, $\eta$ : initial learning rate, $\delta_{r}, \delta_{\eta}$ : decreasing factors for $r$ and $\eta$, respectively, ${ }^{3} k$ : degree of the PSN, $P_{k}$ at the $k$ th algorithmic step, $t$ : number of training epochs, $t_{t h}$ : threshold epoch to finish the training, $\hat{P}_{k}: P_{k}$ after its weights are frozen, and $y_{k}$ : network output at

\footnotetext{
${ }^{3}$ These parameters are needed because the dynamic range of error becomes smaller with successive addition of higher-degree PSN's.
} 
the $k$ th algorithmic step, $=\sigma\left(\sum_{i=k_{0}-1}^{k-1} \hat{P}_{i}+P_{k}\right)$.

\section{Constructive Learning Algorithm for RPN}

1) Initialization Step: Set $k=k_{0}(\geq 1), \hat{P}_{k_{0}-1}=0$ and $t=0$. Assign suitable values to $\epsilon_{t h}, \epsilon_{p}, r, \eta, \delta_{r}, \delta_{\eta}$ and $t_{t h}$.

2) Set $P_{k}(\mathbf{x})=\prod_{j=1}^{k}\left(\left\langle\mathbf{w}_{j}, \mathbf{x}\right\rangle+w_{j 0}\right)$.

3) For all training patterns do:

a) Calculate actual network output, $y_{k}$

b) Update weights by applying the asynchronous update rule as

$$
\begin{gathered}
\Delta \mathbf{v}_{l}=\eta \cdot\left(f-y_{k}\right) \cdot y_{k}^{\prime} \cdot\left(\prod_{j \neq l}^{k}\left(\left\langle\mathbf{w}_{j}, \mathbf{x}\right\rangle+w_{j 0}\right)\right) \cdot \mathbf{z} \\
(l=1, \cdots, k)
\end{gathered}
$$

where $\mathbf{z}=(1, \mathbf{x}), \mathbf{v}_{j}=\left(w_{j 0}, \mathbf{w}_{j}\right) \in \Re^{d+1}$ are augmented input and weight vectors, respectively and $y_{k}^{\prime}$ is the first derivative of the network output.

4) At the end of every epoch (i.e., after applying the above procedure for all the training patterns), calculate the error for the current epoch, $\epsilon_{c}$.

5) If $\epsilon_{c}<\epsilon_{t h}$ or $t>t_{t h}$, stop.

6) Else do:

a) If $\|\left[\left(\epsilon_{c}-\epsilon_{p}\right) / \epsilon_{p} \| \geq r\right.$ do:

- $t \leftarrow t+1$

- $\epsilon_{p} \leftarrow \epsilon_{c}$

- $\quad$ Go to Step 3.

b) Else do:

$$
\begin{array}{ll}
\text { - } & \hat{P}_{k} \leftarrow P_{k} \\
\text { - } & r \leftarrow r \cdot \delta_{r} \\
\text { - } & \eta \leftarrow \eta \cdot \delta_{\eta} \\
\text { - } & t \leftarrow t+1 \\
\text { - } & \epsilon_{p} \leftarrow \epsilon_{c} \\
\text { - } & k \leftarrow k+1 \\
& \text { Go to Step } 2 .
\end{array}
$$

An unknown function $f$ is successively approximated by this incremental learning algorithm as

$$
\begin{aligned}
& f \approx \sigma\left(P_{k_{0}}\right) \\
& f \approx \sigma\left(\hat{P}_{k_{0}}+P_{k_{0}+1}\right), \\
& f \approx \sigma\left(\hat{P}_{k_{0}}+\hat{P}_{k_{0}+1}+P_{k_{0}+2}\right),
\end{aligned}
$$

The number of parameters used in this algorithm is $\mathcal{O}\left(d \cdot k^{2}\right)$ if we start with $k_{0}=1$ and end up with the $k$ th degree PSN. Only the parameters of the most recently added PSN are adapted in a given epoch. This is contrary to the ordinary higher-order networks which adapt all parameters at each epoch.

\section{Simulation Results}

To show the capability of the incremental learning algorithm for the RPN, we performed the following three experiments using the RPN with the constructive learning algorithm:

- Classification of transient oceanic signals,

- Approximation of two-dimensional (2-D) Gabor function (i.e., surface fitting), and

- Realization of a multivariate polynomial.

\section{A. Classification of Transient Oceanic Signals}

The data set used for the experiments consists of 25dimensional feature vectors extracted from short-duration passive sonar signals due to six types of naturally occurring oceanic sources. The signal source type and number of training and testing patterns are given in Table $I$.

Signal preprocessing as well as the extraction of good feature vectors is crucial to the performance of the classifiers [1]. The 25-dimensional feature vectors extracted from the raw signals consist of:

- 16 coefficients of Gabor wavelets-a multiscale representation that does not assume signal stationarity [4],

- One value denoting signal duration, and

- Eight other temporal descriptors and spectral measurements.

The preprocessing techniques and choice of the feature vector reflect the following two goals: i) all effects that vary, but not as a result of the events of interest, be removed or accounted for to the greatest possible extent, and ii) the presence of each type of signal of interest should result in a measurable difference in the observable features.

The following network parameters were used for the results reported in this paper: $\eta=0.1, r=0.0001, \delta_{\eta}=0.8$ and $\delta_{r}=0.1$. This set of parameters was chosen by several trials to yield the best performance on the testing sets. While this compromises the dataset's status as a true test set, it yields limits to how well the network can perform. For fair comparisons, parameters choices for other networks were also made in this fashion. The weights were initialized with uniformly-deviated random numbers in $[-1,1]$. The input vectors were of 25 dimensions and the output vectors were of six dimensions where each output represents one out of six signal types. We used the sigmoid for the output activation function, and let the desired output during training be one for the correct class and zero otherwise. The training started with a second degree PSN and added a third degree PSN at the 200th epoch. Fig. 3 shows classification accuracy for both training and testing sets when training epochs increase. The best result for the testing set was $88.6 \%$ ( 890 hits out of 1005) classification accuracy at the 390th epoch. For training set, the accuracy was $93.8 \%$ (647 hits out of 690 ). We could obtain better results for the training set using a longer training phase and addition of PSN's of degree higher than three, however, the results for the testing set were worse than the one reported above, indicating over-training.

Table II compares the results using the RPN with those of finely-tuned MLP with standard backpropagation algorithm, optimal brain damage (OBD) [23] and cascade-correlation 
TABLE I

Description of Oceanic Data Set Used in the Simulation

\begin{tabular}{|c|l|c|c|}
\hline \multirow{2}{*}{ Class } & \multirow{2}{*}{ Signal Description } & \multicolumn{2}{|c|}{ Number of patterns } \\
\cline { 3 - 4 } & Training & Testing \\
\hline A & Porpoise sound & 116 & 284 \\
\hline B & Ice 1 & 116 & 175 \\
\hline C & Ice 2 & 78 & 39 \\
\hline D & Whale sound 1 & 116 & 129 \\
\hline E & Whale sound 2 & 148 & 251 \\
\hline F & Background noise & 116 & 127 \\
\hline \hline & Totals & $\mathbf{6 0 0}$ & 1005 \\
\hline
\end{tabular}

algorithm [9] reported in [41]. For MLP, OBD, and cascadecorrelation, the classification accuracies for the testing set were obtained after training up to 300 epochs. For these networks, the learning rate was 0.2 for the first 20 epochs and 0.1 after that. The momentum factor was 0.9 , and the initial weights were randomly selected from $[-1,1]$. For the training set, these networks showed quite reasonable results ranging from $90 \%$ to $100 \%$.

We also compare the computational complexity of all the networks used in terms of the number of multiplications needed to finish the training, which has the same order as the number of additions. To obtain these numbers, we first note that each iteration, involving presentation of one training pattern, largely consists of two phases: i) the forward phase involving calculation of network outputs and ii) the backward phase for updating the weights. Thus, number of multiplications needed to finish the training can be obtained by multiplying number of multiplications per iteration by the number of training patterns and the number of epochs to finish the training. In this way, the comparison of each network's computational complexity can be done in a more equitable fashion, and with less dependence on the simulation environment. The calculation of error after each epoch, which is common to all three networks, is not considered. Also, we ignore the computation of the activation function, but note that this simplification favors the MLP type networks as they use a nonlinear activation function for the hidden units while the RPN does not.

As can be observed, the RPN achieved results comparable to other networks, but used much less computation and memory (number of units, weights). Note that we do not have to determine a priori the structure of the RPN as opposed to MLP and OBD in which we should first determine the number of hidden units. The cascade-correlation algorithm, which is a constructive method avoids this problem, however, simulation results suggests that this algorithm sometimes overfits the problem (the classification accuracy of this algorithm for the training set was $100 \%$ using an average of 27.4 hidden units), and shows a wider variation in performance.

\section{B. Multivariate Function Approximation}

In this experiment, we aimed at observing how smooth RPN can generalize using a set of sparse data from an unknown multivariate function. A 2-D Gabor function was tried for this purpose. It is well known that the highlyoriented simple cell receptive fields in the visual cortex of mammals can be closely modeled by 2-D Gabor functions,
TABLE II

Comparative Results for Classification of OcEanic Signals Using the RPN, MLP WITH STANDARD BACKPROPAGATION ALGORITHM, OBD AND Cascade-Correlation Algorithm. For OBD, "Saliency" Was Calculated at Every 10 Epochs. The Number of Hidden Units in the CASCADE - Correlation Algorithm Is the AVERAGE of 8 RUNS

\begin{tabular}{|l|c|c|c|}
\hline Networks & Epochs & $\begin{array}{c}\text { Testing set correct } \\
\text { classification rate }\end{array}$ & \# Multiplications $\left(\times 10^{6}\right)$ \\
\hline \hline RPN & & & \\
2nd order & 50 & 84.1 & 31.9 \\
2nd order & 100 & 84.8 & 63.8 \\
3rd order & 200 & 83.6 & 191.7 \\
3rd order & 300 & 87.8 & 319.6 \\
3rd order & 390 & 88.6 & 434.7 \\
\hline \hline Backpropagation & & & \\
10 hidden units & 300 & 84.0 & 333.3 \\
20 hidden units & 300 & 89.2 & 666.6 \\
40 hidden units & 300 & 90.5 & 1333.1 \\
80 hidden units & 300 & 91.0 & 2666.2 \\
\hline \hline OBD & & & \\
10 hidden units & 300 & 86.2 & 401.2 \\
20 hidden units & 300 & 89.3 & 802.3 \\
40 hidden units & 300 & 90.1 & 1604.7 \\
80 hidden units & 300 & 89.7 & 3209.3 \\
\hline \hline Cascade-correlation & & & \\
23.2 hidden units & 300 & 88.3 & 221.2 \\
27.4 hidden units & 300 & 85.9 & 267.6 \\
\hline
\end{tabular}

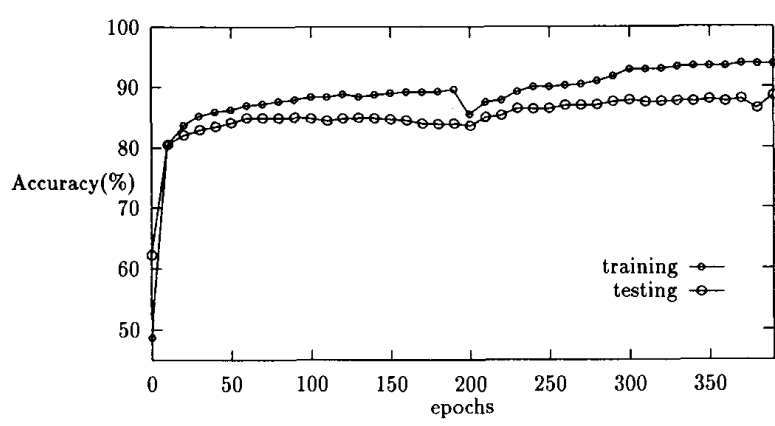

Fig. 3. Classification accuracy for oceanic transient data using the RPN.

which are Gaussians modulated by sinusoidal functions. Gabor functions play an important role in physics, since they are the unique functions that achieve the lower bound for the space-frequency uncertainty product, which is a measure of a function's simultaneous localization in both spatial and frequency domains [15]. The convolution version of complex 2-D Gabor functions have the following form

$$
\begin{aligned}
h\left(x_{1}, x_{2}\right)= & \frac{1}{2 \pi \lambda \sigma^{2}} \cdot e^{-\left\{\left[\left(x_{1} / \lambda\right)^{2}+x_{2}^{2}\right] / 2 \sigma^{2}\right\}} \\
& \cdot e^{2 \pi i\left(u_{0} x_{1}+v_{0} x_{2}\right)}
\end{aligned}
$$

Here, $\lambda$ is the aspect ratio, $\sigma$ is the scale factor and $\left(u_{0}, v_{0}\right)$ are modulation parameters. For simulation, the Gabor function to be approximated was

$$
\begin{aligned}
h\left(x_{1}, x_{2}\right)= & \frac{1}{2 \pi(0.5)^{2}} \cdot e^{-\left[\left(x_{1}^{2}+x_{2}^{2}\right) / 2(0.5)^{2}\right]} \\
& \cdot \cos \left(2 \pi\left(x_{1}+x_{2}\right)\right)
\end{aligned}
$$

Fig. 4(a) shows the Gabor function used for the simulation as desired output. For training, 128 input points were randomly selected from 256 input points taken from an evenly spaced $16 \times 16$ grid on $[-0.5,0.5]^{2}$. The remaining 128 points 


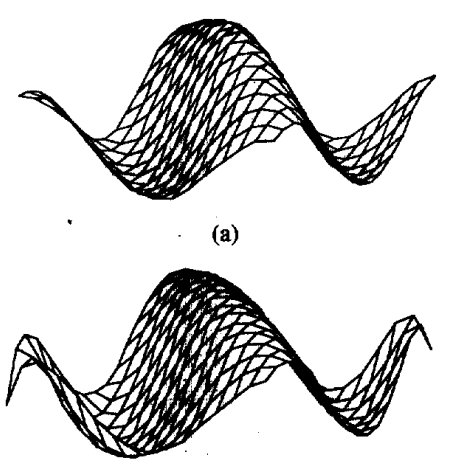

(b)

Fig. 4. Approximation results: (a) Gabor function to be approximated, (b) output of the RPN.

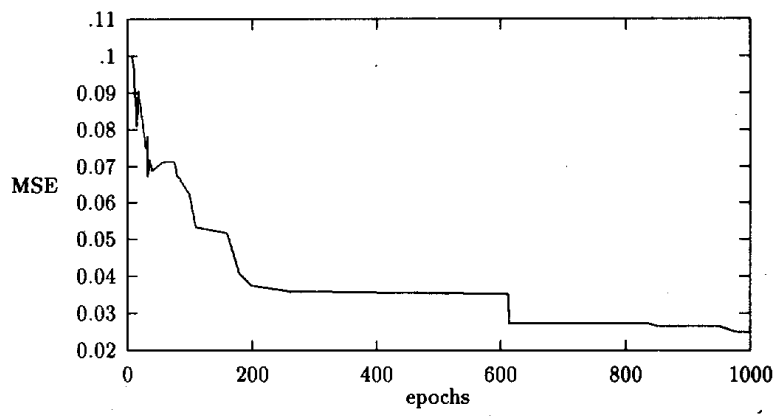

Fig. 5. The learning curve for the approximation of Gabor function using the incremental learning algorithm for the RPN (up to 1000 epochs). Starting with a fourth degree PSN, the algorithm added the fifth and sixth degree PSN's at the 76th and 613th epoch, respectively.

were used for testing. The RPN used a hyperbolic tangent activation function $\sigma(\cdot)=\tanh (\cdot)$ as an output activation function for smoother generalization. All the initial weights used were randomly assigned to values between -0.5 and 0.5. The parameters $\eta, r, \delta_{\eta}$ and $\delta_{r}$ were set to $0.4,0.0$ $001,0.6$ and 0.1 , respectively. The RPN started the training with a fourth degree PSN, and the algorithm added the fifth and sixth degree PSN's at 76th and 613th epochs, respectively. The learning was quite stable and the MSE decreased when additional higher degree PSN's were co-opted, as shown in Fig. 5. Fig. 4(b) shows the actual network output of an RPN including up to eighth degree PSN's after 10000 epochs. MSE's obtained are 0.003 and 0.004 for the training and testing patterns, respectively. We observe that the RPN generalizes well and achieves a smooth interpolation among the training data points.

The Gabor function has been previously used in [16] to compare the Pi-Sigma network with an MLP. After experimenting with different numbers of hidden units and learning parameters, the best result for a two-layered MLP was obtained using 20 hidden units, with $\nu=0.1$ and $\alpha=0.9$. This configuration yielded an MSE of 0.012 for both training and test sets after 500 epochs. A sixth order HPU, in comparison, yielded MSE (training) of 0.028 and MSE (testing) of 0.037 after 5000 epochs.

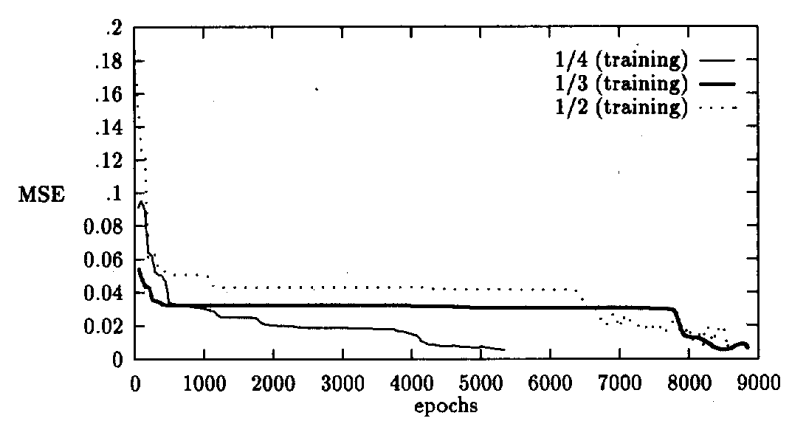

(a)

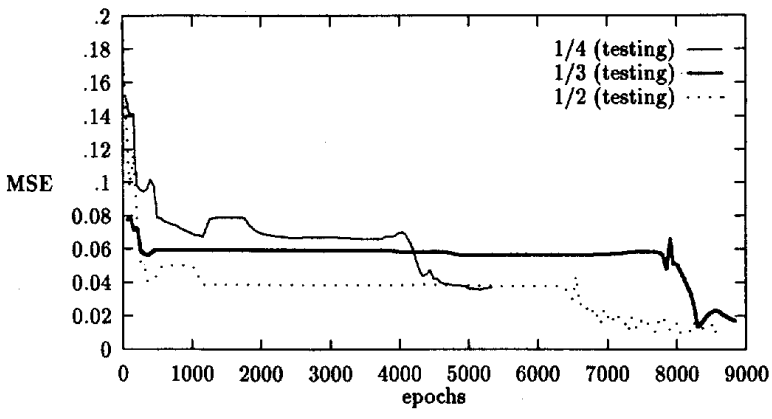

(b)

Fig. 6. The learning curves for the approximation of Gabor function, using the training sets of different sizes: (a) MSE's for the training sets, (b) MSE's for the testing sets.

TABLE III

SIMULATION RESULtS USING THE RPN FOR THE GABOR FunCTION ApProximation wITH Different Stzes of the Training SET

\begin{tabular}{|c|c|c|c|}
\hline \multirow{2}{*}{} & \multicolumn{3}{|c|}{ Size of the training get } \\
\cline { 2 - 4 } & $1 / 4$ & $1 / 3$ & $1 / 2$ \\
\hline \hline & 0.4 & 0.3 & 0.12 \\
\hline$r$ & 0.005 & 0.005 & 0.005 \\
\hline$\delta_{\eta}$ & 0.6 & 0.8 & 0.9 \\
\hline$\delta_{r}$ & 0.1 & 0.1 & 0.1 \\
\hline $\begin{array}{c}\text { Degree of } \\
\text { initial PSN }\end{array}$ & 4 & 4 & 4 \\
\hline $\begin{array}{c}\text { Degree of } \\
\text { final PSN }\end{array}$ & 7 & 8 & 9 \\
\hline MSE(training) & 0.005 & 0.005 & 0.008 \\
\hline MSE(testing) & 0.037 & 0.016 & 0.008 \\
\hline \multicolumn{4}{|c}{} \\
\hline
\end{tabular}

To see the effect of the size of the training set, we have also tried various combinations of training/testing sets. We used a slightly different parameter value for $\sigma$, where the scale factor $\sigma$ in the Gabor function was set to 0.42 rather than 0.5 as used in the previous simulation set (20). Using smaller $\sigma$ increases the dynamic range of the function. Out of 256 input points, we chose $1 / 4,1 / 3$, and $1 / 2$ of 256 points as training set, corresponding to 64,85 , and 128 training points, respectively. As expected, the generalization capability indicated by the approximation accuracy increases for the testing set as more points are included in the training set, while it becomes worse for the training set. Table 3 summarizes the results. Figs. 6(a) and (b) show the learning curves for both training and testing sets for all three cases using the RPN.

To see how the algorithm performs for noisy data, we added noise to the training set. We chose one-fourth out of 256 
TABLE IV

Simulation Results Using the RPN for the Gabor Function approximation with Different Noise Levels on the Training Set

\begin{tabular}{|c|c|c|c|}
\hline \multirow{2}{*}{} & \multicolumn{3}{|c|}{ Noise (\%) } \\
\cline { 2 - 4 } & $0 \%$ & $10 \%$ & $20 \%$ \\
\hline \hline$\eta$ & 0.4 & 0.3 & 0.12 \\
\hline$r$ & 0.005 & 0.005 & 0.005 \\
\hline$\delta_{\eta}$ & 0.6 & 0.8 & 0.98 \\
\hline$\delta_{r}$ & 0.1 & 0.1 & 0.1 \\
\hline $\begin{array}{c}\text { Degree of } \\
\text { initial PSN }\end{array}$ & 4 & 4 & 4 \\
\hline $\begin{array}{c}\text { Degree of } \\
\text { final PSN }\end{array}$ & 7 & 10 & 6 \\
\hline MSE(training) & 0.005 & 0.009 & 0.02 \\
\hline MSE(testing) & 0.037 & 0.027 & 0.04 \\
\hline
\end{tabular}

input points as the training set as in the previous simulation. Then we added uniformly-deviated random noise to all these 64 training points. Testing data had no noise. The first case was adding noise from $[-0.03,0.03]$ and the second case was adding noise from $[-0.06,0.06]$. The maximum values $(0.03$ and 0.06 ) are roughly corresponding to $10 \%$ and $20 \%$ of the average values of all training patterns, respectively. Table IV shows the simulation results. We observe that with higher level of noise, the approximation accuracy for the training set becomes worse. However, slight addition of noise (10\% case) increased the generalization capability. Figs. 7(a) and (b) show the learning curves for both training and testing sets for various noise levels using the RPN.

\section{Realization of Multivariate Polynomial}

Finally, the RPN was trained to realize a multivariate polynomial $f:[-1,1]^{5} \rightarrow \Re$ which was given by ${ }^{4}$

$$
f\left(x_{1}, x_{2}, x_{3}, x_{4}, x_{5}\right)=2+3 x_{1} x_{2}+4 x_{3} x_{4} x_{5} .
$$

Unlike the other two classification/regression problems considered, this simulation mainly focuses on the realization of an unknown polynomial since the RPN uses special forms of polynomials. Note that we do not expect exact representation because of the limited number of training data and the nature of the least square learning used in the incremental learning algorithms.

We randomly selected 200 input points from $[-1,1]^{5}$ and used them as a training set. The weights were initialized by uniformly-deviated random numbers in $[-1,1]$. The parameters $\eta, r, \delta_{\eta}$, and $\delta_{r}$ were set to $0.02,0.0001,0.8$ and 0.1 , respectively. Since the emphasis was on the possibility of the representation, we did not have a testing set to test generalization. The RPN was trained starting from the 1st degree initial PSN (i.e., linear perceptron) with linear output activation function.

The second, third and fourth degree PSN's were added at sixth, 22nd and 65th epochs, respectively, and the training finished at 238th epoch with MSE of 0.02 . The final polynomial $\hat{f}$ realized by the RPN after training can be expanded as an ordinary multivariate polynomial as follows

$$
\begin{aligned}
& \hat{f}\left(x_{1}, x_{2}, x_{3}, x_{4}, x_{5}\right) \approx 1.96+3.03 x_{1} x_{2}+3.74 x_{3} x_{4} x_{5} . \\
& { }^{4} \text { This polynomial was studied in [33]. }
\end{aligned}
$$

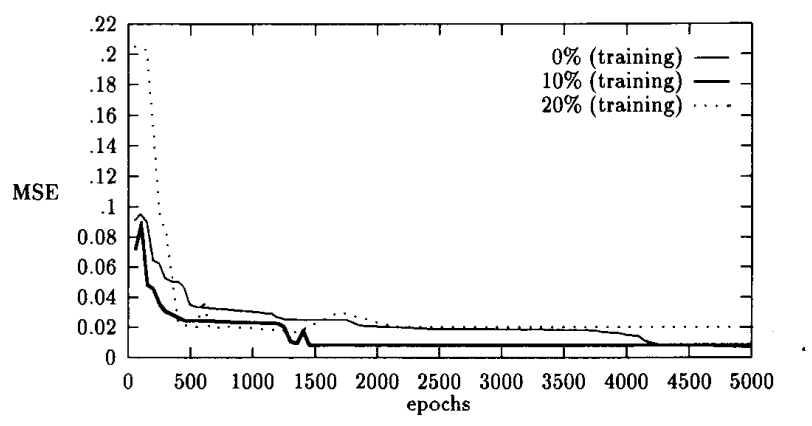

(a)

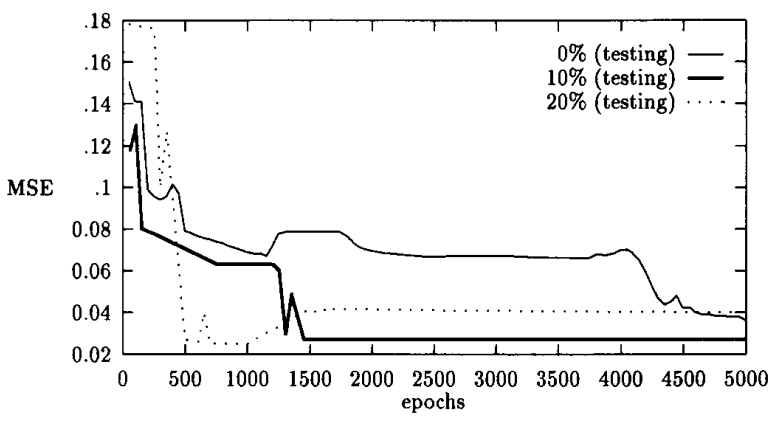

(b)

Fig. 7. The learning curves for the approximation of Gabor function, using the training sets with different noise levels: (a) MSE's for the training sets, (b) MSE's for the testing sets.

Fig. 8 shows all 126 coefficients which were obtained by the RPN after expansion. Note that 126 coefficients correspond to all possible coefficients for fourth degree (ordinary) multivariate polynomial with five inputs (see (2)). The largest unnecessary coefficient was 0.27 for the $x_{3}^{2} x_{4}$ term. This is below $10 \%$ of the smallest coefficient used in $f$ (constant term 2). Note that although the algorithm added fourth degree PSN even though the highest order of $f$ is three, the fourth order terms obtained by the algorithm have negligible magnitudes. Also the representation achieved is very close to the original function $f$ as given in (22), which demonstrates the capability of the algorithm even with a small number of training points. To see how incremental learning achieves better approximation through training, Fig. 9 shows coefficients of up to third degree PSN's at the 64th epoch, i.e., just before the fourth degree PSN was added. There are the total of 56 coefficients after expansion. Using PSN's of degrees up to three, the realized function $\hat{f}$ was

$$
\begin{aligned}
\hat{f}\left(x_{1}, x_{2}, x_{3}, x_{4}, x_{5}\right) \approx & 1.76+2.77 x_{1} x_{2} \\
& +0.53 x_{1} x_{3}+3.45 x_{3} x_{4} x_{5} .
\end{aligned}
$$

As can be observed, addition of higher degree PSN rendered closer approximation to the function $f$ to be realized. Fig. 10 demonstrates stable learning during the training for the RPN.

\section{CONCLUDING REMARKS}

The ridge polynomial network (RPN) is based on a generalization of both Chui and Li's multivariate polynomial 


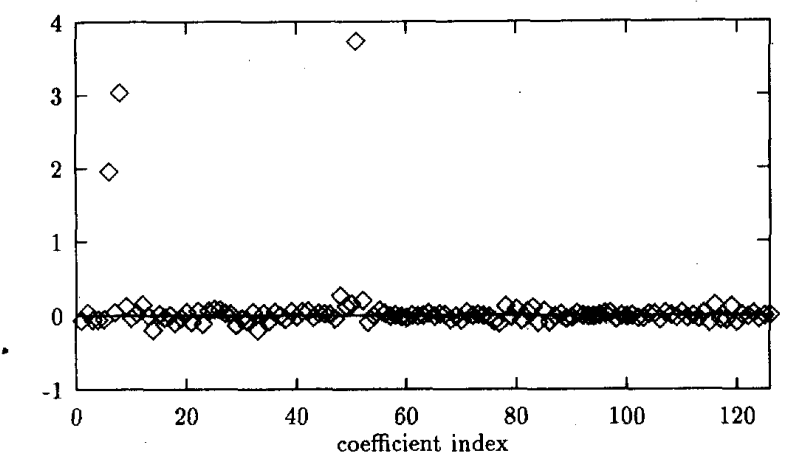

Fig. 8. The coefficients obtained by the RPN of degree up to four. The "coefficient index" just indicates each coefficient out of the total of 126 coefficients after expanding the RPN. The sixth, eighth, and 51st indexes correspond to constant, $x_{1} x_{2}$ and $x_{3} x_{4} x_{5}$ terms, respectively.

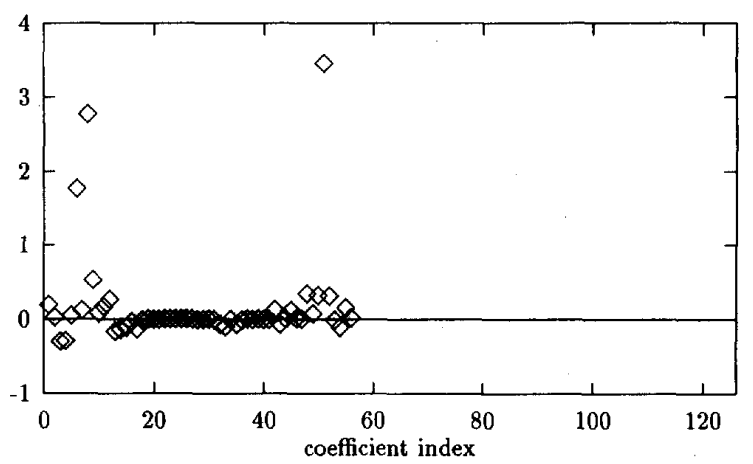

Fig. 9. The coefficients obtained by the RPN of degree up to three. There are the total of 56 coefficients after expanding the RPN. As in the case of fourth degree RPN, the sixth, eighth and 51st indexes correspond to constant, $x_{1} x_{2}$ and $x_{3} x_{4} x_{5}$ terms, respectively.

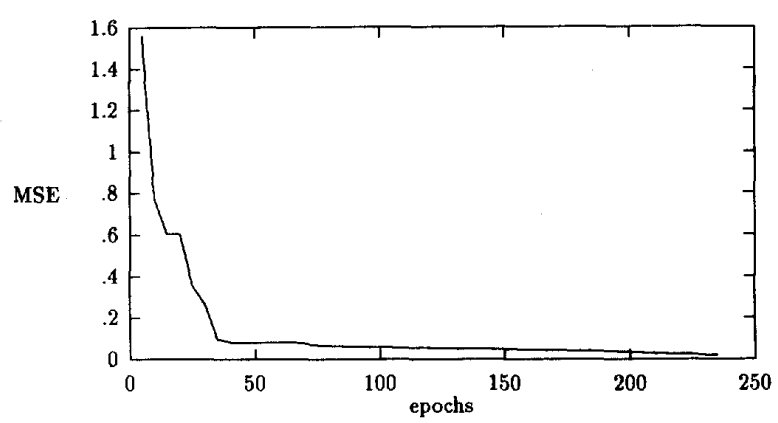

Fig. 10. The learning curve for the realization of multivariate polynomial using the RPN.

representation theorem [3] and the pi-sigma network [16], [34], [35]. We consider it as a special form of projection pursuit regression that is amenable to adaptive, incremental, supervised learning.

Simulation results on a surface fitting problem show that the constructive learning algorithm proposed for the RPN produces a smooth generalization which is a desirable property in multivariate function approximation. Successive incorporation of higher-order terms during learning captures the higher-order variations in the problem. A key feature of RPN network growth is that it takes place naturally as part of the training process, while maintaining a regular structure. It is widely acknowledged that there is no single method-statistical or neural--that gives the best results for all function approximation problems [11]. Thus, while our simulations demonstrate several desirable properties of RPN's, a deeper understanding of the classes of functions for which they are natural can only be obtained after extensive comparative experiments with several different techniques and data sets.

The difficult theoretical question of whether the constructive learning algorithm can achieve the same or similar approximation capability as an adequately large fixed structure is left as future work. Also, optimization techniques like "orthogonalization" of polynomial bases can be investigated to improve the performance of the networks without substantially increasing their complexity.

\section{APPENDIX A}

\section{PROOF OF THEOREM 2}

Proof: We want to show that right-hand side of (7) can be expressed as a special case of right-hand side of (8). From (7)

$$
\begin{aligned}
\sum_{j=0}^{k} \sum_{m=1}^{n_{j}} a_{j m}\left\langle\mathbf{x}, \mathbf{v}_{j m}\right\rangle^{j}= & \sum_{m=1}^{n_{0}} a_{0 m}+\sum_{m=1}^{n_{1}} a_{1 m}\left\langle\mathbf{x}, \mathbf{v}_{1 m}\right\rangle \\
& +\cdots+\sum_{m=1}^{n_{k}} a_{k m}\left\langle\mathbf{x}, \mathbf{v}_{k m}\right\rangle^{k} \\
= & \sum_{l=0}^{k} p_{l}(\mathbf{x})
\end{aligned}
$$

where

$$
\begin{gathered}
p_{0}(\mathbf{x})=\sum_{m=1}^{n_{0}}\left(\langle\mathbf{x}, \mathbf{0}\rangle+a_{0 m}\right)(\langle\mathbf{x}, \mathbf{0}\rangle+1)^{m-1}, \\
p_{l}(\mathbf{x})=\sum_{m=1}^{n_{l}}\left(\left\langle\mathbf{x}, a_{l m} \mathbf{v}_{l m}\right\rangle+0\right)\left(\left\langle\mathbf{x}, \mathbf{v}_{l m}\right\rangle+0\right)^{l-1} \\
\cdot(\langle\mathbf{x}, \mathbf{0}\rangle+1)^{n_{0}+\cdots+n_{l-1}+m-l}, \\
\quad l=1, \cdots, k
\end{gathered}
$$

$\mathbf{x}, \mathbf{v}_{j m} \in \Re^{d}, a_{j m} \in \Re, a_{j m} \mathbf{v}_{j m}=\left(a_{j m} v_{j m 1}, \cdots, a_{j m} v_{j m d}\right) \in$ $\Re^{d}$, and $\mathbf{0}=(0, \cdots, 0) \in \Re^{d}$. By proper change of variables $\left(j=m\right.$ for $p_{0}(\mathbf{x})$ and $j=n_{0}+\cdots+n_{l-1}+m$ for $\left.p_{l}(\mathbf{x}), l=1, \cdots, k\right)$, (24) becomes

$$
\begin{aligned}
\sum_{j=0}^{k} \sum_{m=1}^{n_{j}} a_{j m}\left\langle\mathbf{x}, \mathbf{v}_{j m}\right\rangle^{j}= & \sum_{l=0}^{k} p_{l}(\mathbf{x}) \\
= & \sum_{j=1}^{n_{0}}\left(\langle\mathbf{x}, \mathbf{0}\rangle+a_{0 j}\right)(\langle\mathbf{x}, \mathbf{0}\rangle+1)^{j-1} \\
& +\sum_{j=n_{0}+1}^{n_{0}+n_{1}}\left(\left\langle\mathbf{x}, a_{1, j-n_{0}} \mathbf{v}_{1, j-n_{0}}\right\rangle+0\right) \\
& \cdot(\langle\mathbf{x}, \mathbf{0}\rangle+1)^{j-1}
\end{aligned}
$$




$$
\begin{aligned}
& +\sum_{j=n_{0}+\cdots+n_{k-1}+1}^{n_{0}+\cdots+n_{k}} \\
& \cdot\left(\left\langle\mathbf{x}, a_{k, j-\left(n_{0}+\cdots+n_{k-1}\right)}\right.\right. \\
& \left.\left.\cdot \mathbf{v}_{k, j-\left(n_{0}+\cdots+n_{k-1}\right)}\right\rangle+0\right) \\
& \cdot\left(\left\langle\mathbf{x}, \mathbf{v}_{k, j-\left(n_{0}+\cdots+n_{k-1}\right)}\right\rangle+0\right)^{k-1} \\
& \cdot(\langle\mathbf{x}, 0\rangle+1)^{j-k} .
\end{aligned}
$$

Also from (8)

$$
\begin{aligned}
\sum_{j=1}^{N} \prod_{i=1}^{j}\left(\left\langle\mathbf{x}, \mathbf{w}_{j i}\right\rangle+w_{j i}\right)= & \sum_{j=1}^{n_{0}} \prod_{i=1}^{j}\left(\left\langle\mathbf{x}, \mathbf{w}_{j i}\right\rangle+w_{j i}\right) \\
& +\sum_{j=n_{0}+1}^{n_{0}+n_{1}} \prod_{i=1}^{j} \\
& \cdot\left(\left\langle\mathbf{x}, \mathbf{w}_{j i}\right\rangle+w_{j i}\right)+\cdots \\
& +\sum_{j=n_{0}+\cdots+n_{k-1}+1}^{n_{0}+\cdots+n_{k}} \prod_{i=1}^{j} \\
& \cdot\left(\left\langle\mathbf{x}, \mathbf{w}_{j i}\right\rangle+w_{j i}\right) .
\end{aligned}
$$

Since (27) is a special case of (28), this completes the proof.

\section{APPENDIX B \\ GENERALIZED WEIERSTRASS \\ POLYNOMIAL APPROXIMATION THEOREM}

The well-known Weierstrass polynomial approximation theorem for univariate continuous functions can be generalized to multidimensional settings. This generalized theorem specifies the uniform approximation capability of polynomials.

Theorem 3 (Generalized Weierstrass Polynomial Approximation Theorem [7]): Let $K \subset \Re^{d}(d \geq 1)$ be compact. Then for any function $f \in C(K)$, the vector space of continuous scalar functions on $K$, there exists a polynomial $p \in C(K)$ such that for given $\epsilon>0,|f(\mathbf{x})-p(\mathbf{x})|<\epsilon$ for all $\mathbf{x} \in K$.

For hypercubes $[0,1]^{d}$, the generalized multivariate Bernstein polynomials [7] are shown to have the property mentioned in the theorem.

The theorem stated above can be obtained as a corollary of the Stone-Weierstrass theorem.

Theorem 4 (Stone-Weierstrass Theorem [31]): Let $K \subset$ $\Re^{d}(d \geq 1)$ be compact and $A$ be an algebra of continuous scalar functions on $K$ which separates the points in $K$ and which contains the constant functions. Then $A$ is dense in $C(K)$, the vector space of continuous scalar functions on $K$.

A subspace $A$ of $C(K)$ is an algebra if whenever $f, g \in A$, then $f \cdot g \in A$. An algebra $A$ separates the points in $K$ if for any $\mathbf{x}, \mathbf{y} \in K$ and $\mathbf{x} \neq \mathbf{y}$, there exists a function $f \in A$ such that $f(\mathbf{x}) \neq f(\mathbf{y})$. The generalized Weierstrass polynomial approximation theorem follows from this theorem since the family of polynomials is an algebra which satisfies all the conditions of the theorem.

\section{ACKNOWLEDGMENT}

The authors wish to thank S. Im for preparing surface plots used in this paper and $\mathrm{K}$. Tumer for providing simulation results of the multilayered perceptron, optimal brain damage, and cascade-correlation algorithm on the oceanic signals classification, as well as the anonymous reviewers for helpful comments.

\section{REFERENCES}

[1] S. Beck, L. Deuser, R. Still, and J. Whiteley, "A hybrid neural network classifier of short duration acoustic signals," in Proc. Int. Joint Conf. Neural Networks, vol. I, Seattle, WA, July 1991, pp. 119-124.

[2] H. H. Chen, Y. C. Lee, T. Maxwell, G. Z. Sun, H. Y. Lee, and C. L Giles,"Higher order correlation model for associative memory," in AIP Conf. Proc. 151,1986, pp. 86-99.

[3] C. K. Chui and X. Li, "Realization of neural networks with one hidden layer," Center for Approximation Theory, Dept. of Mathematics, Texas A\&M Univ., Tech. Rep. \#CAT-244, Mar. 1991.

[4] J. M. Combes, A. Grossman, and P. Tchamitchian, Eds., Wavelets: TimeFrequency Methods and Phase Space. New York: Springer-Verlag, 1989.

[5] N. E. Cotter, "The Stone-Weierstrass theorem and its application to neural networks," IEEE Trans. Neural Networks, vol. 1, no. 4, pp. $290-295,1990$.

[6] G. Cybenko, "Approximations by superposition of a sigmoidal function,"Math. Contr., Signals, Sys., vol. 2, pp. 303-314, 1989.

[7] P. J. Davis, Interpolation and Approximation. New York: Dover, 1975

[8] R. Durbin and D. E. Rumelhart, "Product units: A computationally powerful and biologically plausible extension to backpropagation networks," Neural Computation, vol. 1, pp. 133-142, 1989.

[9] S. E. Fahlman and C. Lebiere, "The cascade-correlation learning architecture," in Advances in Neural Information Processing Systems 2 D. S. Touretzky, Ed. San Mateo, CA: Morgan-Kaufmann, 1990, pp. 525-532

[10] J. A. Feldman and D. H. Ballard, "Connectionist models and their properties," Cognitive Science, vol. 6, pp. 205-254, 1982.

[11] J. H. Friedman, "Adaptive techniques for machine learning and function approximation," in From Statistics to Neural Networks, presented at NATO ASI Workshop, Les Arcs, France, June 1993.

[12] J. H. Friedman and W. Stuetzle, "Projection pursuit regression,"Journal of the Amer. Stat. Assoc., vol. 76, no. 376, pp. 817-823, Dec. 1981.

[13] J. H. Friedman, W. Stuetzle, and A. Schroeder, "Projection pursuit density estimation," J. Amer. Statist. Ass., vol. 79, no. 387, pp. 599-608, Sept. 1984.

[14] S. Geman, E. Bienenstock, and R. Doursat, "Neural networks and the bias/variance dilemma," Neural Computation, vol. 4, no. 1, pp. 1-58, 1992.

[15] J. Ghosh and A. C. Bovik, "Neural networks for textured image processing," in Artificial Neural Networks and Statistical Pattern Recognition. Old and New Connections, I. K. Sethi and A. K. Jain, Eds. Amsterdam, The Netherlands: Elsevier, 1991, pp. 133-154.

[16] J. Ghosh and Y. Shin, "Efficient higher-order neural networks for classification and function approximation," Int. J. Neural Syst., vol. 3, no. 4, pp. 323-350, 1992

[17] C. L. Giles and T. Maxwell, "Learning, invariance, and generalization in a high-order neural network," Applied Optics, vol. 26, no. 23, pp 4972-4978, 1987.

[18] K. Hornik, M. Stinchcombe, and H. White, "Multilayer feedforward networks are universal approximators," Neural Networks, vol. 2, pp. 359-366, 1989.

[19] J.-N. Hwang, H. Li, M. Maechler, R. D. Martin, and J. Schimert, "A comparison of projection pursuit and neural network regression modeling," in Advances in Neural Information Processing Systems 4, S. J. Hanson, J. E. Moody, and R. P. Lippmann, Eds. San Mateo, CA: Morgan-Kaufmann, 1992, pp. 1159-1166.

[20] P. J. Huber, "Projection pursuit," Annals Statis.,vol. 12, pp. 435-475, 1985.

[21] A. G. Ivakhnenko, "Polynomial theory of complex systems," IEEE Trans. Syst., Man, Cybern., vol. 1, no. 4, pp. 364-378, Oct. 1971

[22] C. Koch and T. Poggio, "Multiplying with synapses and neurons," in Single Neuron Computation, T. McKenna, J. Davis, and S. F. Zornetzer, Eds. New York: Academic, 1992.

[23] Y. LeCun, J. S. Denker, and S. A. Solla, "Optimal brain damage," in Advances in Neural Information Processing Systems 2, D. S. Touretzky, Ed. San Mateo, CA: Morgan-Kaufmann, 1990, pp. 598-605. 
[24] M. R. Lynch and P. J. Rayner, "The properties and implementation of the nonlinear vector space connectionist model," in Proc. 1st IEE Int. Conf. Art. Neural Networks, London, England, Oct. 1989, pp. 184-190.

[25] J. McClelland and D. Rumelhart, Parallel Distributed Processing, Vol. 1. Cambridge, MA: MIT Press, 1987.

[26] B. W. Mel, "The clusteron: Toward a simple abstraction for a complex neuron," in Advances in Neural Information Processing Systems 4, S. J. Hanson, J. E. Moody, and R. P. Lippmann, Eds. San Mateo, CA: Morgan-Kaufmann, 1992, pp. 35-42.

[27] B. W. Mel and C. Koch."Sigma-pi learning: On radial basis functions and cortical associative learning," in Advances in Neural Information Processing Systems 2, D. S. Touretzky, Ed. San Mateo, CA: MorganKaufmann, 1990, pp. 474-481.

[28] Y. Pao, Adaptive Pattern Recognition and Neural Networks. Reading, MA: Addison-Wesley, 1989.

[29] J. Park and I. W. Sandberg, "Universal approximation using radialbasis-function networks," Neural Computation, vol. 3, pp. 246-257, 1991.

[30] D. Psaltis, C. H. Park, and J. Hong, "Higher order associative memories and their optical implementation," IEEE Trans. Neural Networks, vol. 1, pp. $149-163,1988$.

[31] H. L. Royden, Real Analysis, 2nd Ed. New York: MacMillan, 1968.

[32] T. D. Sanger "A tree-structured adaptive network for function approximation in high-dimensional spaces," IEEE Trans. Neural Networks, vol. 2, no. 2, Mar. 1991.

[33] T. D. Sanger, R. S. Sutton, and C. J. Matheus, "Iterative construction of sparse polynomial approximations," in Advances in Neural Information Processing Systems 4, S. J. Hanson, J. E. Moody, and R. P. Lippmann, Eds. San Mateo, CA: Motgan-Kaufmann, 1992, pp. 1064-1071.

[34] Y. Shin and J. Ghosh, "The pi-sigma network: An efficient higher-order neural network for pattern classification and function approximation," in Proc. Int. Joint Conf. Neural Networks, Seattle, WA, July 1991, vol I, pp. 13-18.

[35] works," in Intelligent Engineering Systems through Artificial Neural Networks, C. H. Dagli, S. R. T. Kumara, and Y. C. Shin, Eds. New York: ASME Press, 1991, pp. 205-210, 1991.

[36] $\longrightarrow$, "Approximation of multivariate functions using ridge polynomial networks," in Proc. Int. Joint Conf. Neural Networks, Baltimore, MD, June 1992, vol. II, pp. 380-385.

[37] $\ldots$,Punction approximation using higher-order conmectionist networks," Computer and Vision Research Center, The Univ, of Texas at Austin, TX, Tech. Rep. \#TR-92-12-87, May 1992.

[38] Y. Shin, J. Ghosh, and D. Samani, "Computationally efficient invariant pattem classification with higher-order pi-sigma networks," in Intelligent Engineering Systems through Artificial Neural Networks, C. H. Dagli, L. I. Burke, and Y. C. Shin, Eds. New York: ASME Press, 1992, vol. 2, pp. 379-384.

[39] D. F. Specht, "Generation of polynomial discriminant functions for pattern recognition," IEEE Trans, on Electron. Comput, vol. EC-16, pp. 308-319, 1967.

[40] M. F. Tenorio and W. Lee, "Self-organizing network for optimum supervised learning," IEEE Trans. Neural Networks, vol. 1, no. 1, pp. 100-110, Mar. 1990
[41] K. Tumer, "Structural adaptation and generalization in neural networks," M.S. Thesis, The University of Texas at Austin, Aug. 1992.

[42] S. S. Venkatesh, "Programmed interactions in higher-order neural networks: Maximal capacity," J. Complexity, vol. 7, pp. 316-337, 1991.

[43] M. Vidyasagar, Nonlinear Systems Analysis, 2nd Ed. Englewood Cliffs, NI: Prentice-Hall, 1993.

[44] B. Widrow and S. D. Stearns, Adaptive Signal Processing. Englewood Cliffs, NJ: Prentice-Hall, 1985

[45] Y. Zhao and C. G. Atkeson, "Some approximation properties of projection pursuit learning networks," in Advances in Neural Information Processing Systems 4, S. J. Hanson, J. E. Moody, and R. P. Lippmann, Eds. San Mateo, CA: Morgan-Kaufmann, 1992, pp. 936-943.

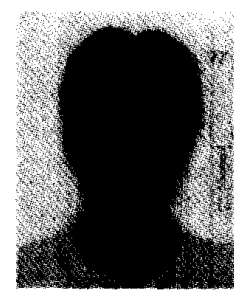

Yoan Shin (S'87-M'90) received the B.S.E.E. and M.E.E. degrees in electronic engineering from Seoul National University, Seoul, Korea, in 1987 and 1989 , respectively, and the Ph.D. degree in electrical and computer engineering at the University of Texas at Austin in 1992.

He was with the Neural Network Research Group, MCC, Austin. He is now an Assistant Professor at Soong Sil University, South Korea. His current research interests include theory and application of artificial neural networks.

Dr. Shin is a recipient of the Overseas Study Fellowship from the Ministry of Education of the Republic of Korea.

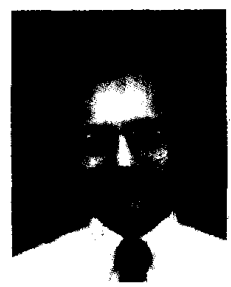

Joydeep Ghosh (M'85) received the M.S. and Ph.D. degrees in computer engineering at the University of Southern California, in 1985 and 1988 , respectively.

$\mathrm{He}$ is an Associate Professor in the Department of Electrical and Computer Engineering at the University of Texas, Austin, TX.

Dr. Ghosh was the first member of the School of Engineering at USC to be awarded an "All University Predoctoral Teaching Fellowship" for four years. He has published over 70 papers on massively parallel processing and on artificial neural systems and their applications in image analysis, signal processing, prediction and control. He is the recipient of the 1992 Darlington Award given by the IEEE Circuits and Systems Society for the Best Journal Paper, and has also received awards for two conference papers on neural networks. He is an associate editor of Pattern Recognition, and a Conference Co-Chair for ANNIE '93 and ANNIE '94. 